\title{
Comparative genomics study of polyhydroxyalkanoates (PHA) and ectoine relevant genes from Halomonas sp. TD01 revealed extensive horizontal gene transfer events and co-evolutionary relationships
}

Lei Cai ${ }^{1 \dagger}$, Dan Tan ${ }^{1 \dagger}$, Gulsimay Aibaidula ${ }^{2}$, Xin-Ran Dong ${ }^{3}$, Jin-Chun Chen ${ }^{1}$, Wei-Dong $\operatorname{Tian}^{3^{*}}$ and Guo-Qiang Chen ${ }^{1 *}$

\begin{abstract}
Background: Halophilic bacteria have shown their significance in industrial production of polyhydroxyalkanoates (PHA) and are gaining more attention for genetic engineering modification. Yet, little information on the genomics and PHA related genes from halophilic bacteria have been disclosed so far.

Results: The draft genome of moderately halophilic bacterium, Halomonas sp. TD01, a strain of great potential for industrial production of short-chain-length polyhydroxyalkanoates (PHA), was analyzed through computational methods to reveal the osmoregulation mechanism and the evolutionary relationship of the enzymes relevant to PHA and ectoine syntheses. Genes involved in the metabolism of PHA and osmolytes were annotated and studied in silico. Although PHA synthase, depolymerase, regulator/repressor and phasin were all involved in PHA metabolic pathways, they demonstrated different horizontal gene transfer (HGT) events between the genomes of different strains. In contrast, co-occurrence of ectoine genes in the same genome was more frequently observed, and ectoine genes were more likely under coincidental horizontal gene transfer than PHA related genes. In addition, the adjacent organization of the homologues of PHA synthase phaC1 and PHA granule binding protein phaP was conserved in the strain TD01, which was also observed in some halophiles and non-halophiles exclusively from $\gamma$ proteobacteria. In contrast to haloarchaea, the proteome of Halomonas sp. TD01 did not show obvious inclination towards acidity relative to non-halophilic Escherichia coli MG1655, which signified that Halomonas sp. TD01 preferred the accumulation of organic osmolytes to ions in order to balance the intracellular osmotic pressure with the environment.
\end{abstract}

Conclusions: The accessibility of genome information would facilitate research on the genetic engineering of halophilic bacteria including Halomonas sp. TD01.

Keywords: Halomonas spp., PHB, polyhydroxyalkanoates, osmolytes, genome, PhaC

\footnotetext{
* Correspondence: weidong.tian@fudan.edu.cn; chengq@mail.tsinghua.edu.cn † Contributed equally

'Dept Biological Sciences and Biotechnology, MOE Key Lab. Bioinformatics (\& System Biology), Tsinghua University-Peking University Joint Center for Life Sciences, School of Life Sciences, Tsinghua University, Beijing 100084, China ${ }^{3}$ Institute of Biostatistics, School of Life Sciences, Fudan University, Shanghai, China

Full list of author information is available at the end of the article
} 


\section{Background}

Polyhydroxyalkanoates (PHA) were firstly discovered in prokaryotes as carbon and energy storage materials [1]. They are one of the most promising members of biodegradable polymers, which are considered as environmentally friendly substitutes of petrochemical-derived plastics [2]. Poly-3-hydroxybutyrate (PHB) was the earliest commercially available product. However, poly(3hydroxybutyrate-co-hydroxyvalerate) (PHBV) possessed more favourable thermomechanical properties for wider applications as medical materials (sutures and bonenails/pins), films products (mulch films, shopping bags and compost bags), disposable items (pens and tableware) and packaging materials (especially for food packaging) than PHB [3]. As the market for green plastics has been growing rapidly, demand for a more productive and low cost PHA production process is evident [1]. Although some microorganisms, such as Ralstonia eutropha and genetically engineered Escherichia coli, were thoroughly investigated as PHA industrial producers with high productivity, the research and development of strains and methods with further reduced cost were still necessary for PHA commercialization [3-6].

Halophiles are referred to some organisms, which are able to grow optimally in $5 \%(\mathrm{w} / \mathrm{v})$ and survive in no less than $10 \%$ salt medium $[7,8]$. Halophiles have shown advantages for lowering fermentation costs for PHB and PHBV production, thus gaining attention from many researchers. As early as 1972, PHA granules were detected in a haloarchaea Haloarcula marismortui, and then numerous halophiles were identified as producers to accumulate PHB or PHBV [7]. Halophiles were widely spread in the three domains of life: archaea, bacteria, and eukarya [7]. According to the optimal growth concentration of salt tolerated by cells, they could be roughly divided into two groups, moderate and extreme halophiles [9]. Recently, the application of some halophiles for PHA production and copolymer characterization were evaluated $[4,7,10,11]$. The application of halophiles as PHA producers significantly reduced the costs of fermentation and recovery processes: high salt concentrations were able to minimize the possibility of contamination by non-halophilic microorganisms, and thus the cost and energy consumption for sterilization can be decreased; haloarchaeal cells were able to be lyzed conveniently through osmotic shock treatment with salt-deficient water, and thus the cost for polymers recovery also can be decreased [12]. Moreover, the residual salt in the broth post-fermentation, could be concentrated and recycled, which circumvents environmental problems from the exhaust of waste products and further lowered the production cost [7]. When hydrolyzed whey was used as carbon source, the cost for the production of $\mathrm{P}(3 \mathrm{HB}-\mathrm{co} 3 \mathrm{HV})$ by Haloferax mediterranei was about $30 \%$ lower than that for the production of PHA by recombinant E. coli [7]. Our recent study showed a new isolated halophilic strain termed Halomonas sp. TD01, has great potential for PHA production, accumulating over $80 \mathrm{wt} \%$ PHB under a continuous fermentation process without sterilization [13].

PHA synthases, the crucial enzymes for PHA biosynthesis, have received more attention for elucidating their characteristics in these microorganisms. PHA synthases were classified into four groups according to their substrate specificities and subunits organization [1]. Although class I and II PHA synthases comprise enzymes consisting exclusively of one PhaC subunit, they prefer to synthesize short chain length (3-5 carbon atoms) and medium chain length (6-14 carbon atoms) PHA, respectively. Class III and IV PHA synthases comprise enzymes consisting of two different types of subunits, PhaC and PhaE (III) or PhaR (IV, such as that in Bacillus megaterium) subunits; both of them prefer to utilize $\mathrm{CoA}$ thioesters to produce short chain length PHA [1]. The study on PHA synthases isolated from PHA accumulating halophiles began with the characterization of PhaC from Halopiger aswanensis, which showed some interesting properties including high thermostability, narrow substrate specificity and tolerance against high salt concentration [14]. A novel subclass of PhaCs was proposed through systematic and detailed studies on the PHA synthases from haloarchaea, which were in high similarity with the class III enzymes from bacteria [15]. However, the $H$. mediterranei PhaC subunit was larger, and the PhaE subunit was smaller than its bacterial counterparts [16].

Osmoregulatory mechanisms of halophiles against the high salt concentration condition were typically divided into two strategies: extreme halophiles, i.e. halophilic archaea, were able to absorb $\mathrm{KCl}$ to balance the osmotic pressure discrepancy across the cytoplasmic membrane; most moderate halophiles were able to accumulate osmolytes (compatible solutes), which mainly consisted of organic compounds with low molecular weight, including amino acids, amino acid derivatives, sugars, and other polyols [17]. Organic compounds did not notably disturb the essential metabolism for cell survival, and protected proteins by mitigating detrimental effects of freezing, drying and high temperatures [8]. Ectoine is one of the most widespread osmolytes, which are also well known as commercial protectants for enzymes, DNA and whole cells [18].

In this study, the draft genome of the moderately halophilic bacterium Halomonas sp. TD01 was obtained and analyzed. Several genes relevant to PHA and osmolytes biosynthesis were elucidated and phylogenetically 
analyzed. Moreover, the predicted proteome was analyzed and compared with that of other species. The results provided invaluable clues, not only to the understanding of the evolution and genes transfer, but also to the strategic guidance of the genetic engineering of halophilic Halomonas sp. TD01 for co-production of PHA and ectoine.

\section{Methods}

\section{Strain and genome DNA preparation}

Halomonas sp. TD01 was isolated from a salt lake in Xinjiang, China, and grown optimally in glucose mineral medium under $5 \%(\mathrm{w} / \mathrm{v}) \mathrm{NaCl}$ and $\mathrm{pH} 9.0$ [13]. And it was deposited in China General Microbiological Culture Collection Center (CGMCC No.4353). To construct the insert libraries for sequencing, high-quality total cellular DNA was prepared with the help of the E.Z.N.A. bacterial DNA kit (Omega Bio-Tek Inc. USA).

\section{Sequencing strategy}

Two random genomic DNA libraries with insert sizes of 500 and 2,530 base pairs (bp) were constructed, respectively. The sequencing of these libraries was carried out following the Solexa sequencing protocols (Illumina, Inc. USA) in the Beijing Genomics Institute (BGI, Beijing, China). Eliminating the low-quality results and adapter contamination, raw data was assembled into contigs and scaffolds with SOAPdenovo software (v1.04, BGI, Beijing, China).

\section{Genome annotation}

Glimmer 3.0 software [19] was adopted to predict genes de novo. For annotation, the alignments of predicted proteins against databases, including KEGG [20], COG [21], Swiss-Prot [22], TrEMBL [23] and nr (at NCBI, National Center for Biotechnology Information), were carried out with the program blastall (version 2.2.21) [24]. Genes encoding transfer RNA (tRNA) and their secondary structure were predicted using tRNAscan [25]. Genes encoding ribosomal RNA (rRNA) were predicted through RNAmmer [26] or homologous comparison. Genes encoding other RNA, including microRNA (miRNA), small RNA (sRNA) and small nuclear RNA (snRNA) were predicted by Rfam [27]. Transposons were identified through RepeatMasker and RepeatProteinMasker [28].

After the assembly, global guanine-cytosine composition of the whole non-redundant sequence was calculated with the in-house program (BGI, Beijing, China). The predicted open reading frames (ORFs) inferred by Glimmer were also counted for GC content and length distribution.

\section{Promoter and signal peptide prediction}

The promoter, including $-35,-10$ and transcription start site (TSS) regions, were predicted through Neural
Network Promoter Prediction [29] and Sequence Alignment Kernel [30] methods. The signal peptide for secreted proteins was predicted by SignalP [31].

\section{Multiple sequences alignments and identification of protein motifs}

Multiple alignments of predicted proteins with conserved sequences were performed with Constraint-based Multiple Alignment Tool (COBALT) [32] at NCBI. Conserved motifs were identified by searching sequences against the COG database.

\section{Evolutionary and phylogenetic analysis}

The distances between conserved sequences were calculated from the multiple alignments with ClustalW [33]. Neighbor-joining tree phylograms were constructed, bootstrapped (500 replications), and drawn in MEGA (version 5.03) [34].

With the evolutionary distances, the evolutionary pressures imposed on keeping any two genes in the genome were inspected by computing a Pearson correlation coefficient for any two genes. The missing genes (designated as "ND") were useful in inspecting the co-evolutionary relationships, and usually defined as zero. However, here, the smaller the evolutionary distance, the closer a homologue is to the gene in TD01. To make use of the missing genes, we transformed the evolutionary distances into similarity scores with $\mathrm{e}^{\left(-1^{*} \text { distance }\right)}$ and defined the missing genes (ND) as zero, i.e. a shorter distance relates to an increased similarity score. Then a Pearson correlation coefficient was computed for any two genes using the converted similarity scores.

\section{Calculation of isoelectric point values}

FASTA format protein sequences, either predicted from Halomonas sp. TD01 or retrieved from NCBI were submitted to ExPASy Proteomics server (http://web.expasy. org/compute_pi/). The isoelectric point (pI) distribution of each strain was counted with an interval of 0.2 differences from 3 to 13 .

\section{Accession numbers}

The draft genome assemblies for Halomonas sp.TD01 were submitted into the GenBank database; its accession number was GenBank: AFQW00000000. Other accession numbers of the sequences involved in this study are listed in Table 1 and Additional file 1, Table S1.

\section{Results}

\section{Overview of sequencing and gene prediction}

Sequencing was conducted with Solexa technology. Using SOAPdenovo software, the clean raw data was assembled into 26 scaffolds varying from 511 to 918,836 bp with a total length of 4,092,837 bp. The estimated 
Table 1 Putative PHA and ectoine relevant genes in the genome of Halomonas sp. TD01

\begin{tabular}{|c|c|c|c|c|c|}
\hline & Enzyme & Gene & Accession Number ${ }^{a}$ & Calculated Molecular mass $(\mathrm{kDa})^{\mathbf{b}}$ & Calculated $\mathrm{pl}^{\mathrm{b}}$ \\
\hline \multirow[t]{7}{*}{$\overline{\mathrm{PHA}}$} & Polyhydroxyalkanoate synthase & phaC1 & EGP20415 & 70 & 4.80 \\
\hline & & phaC2 & EGP19504 & 92 & 5.99 \\
\hline & Polyhydroxyalkanoate depolymerase & phaZ1 & EGP20509 & 40 & 7.75 \\
\hline & & phaz2 & EGP18355 & 33 & 4.80 \\
\hline & & phaz3 & EGP19590 & 38 & 4.68 \\
\hline & Phasin & phap & EGP20414 & 16 & 5.64 \\
\hline & Polyhydroxyalkanoate synthesis repressor & phar & EGP21321 & 18 & 4.78 \\
\hline \multirow[t]{4}{*}{ Ectoine } & L-2,4-diaminobutyric acid acetyltransferase & ectA & EGP18461 & 21 & 4.94 \\
\hline & L-2,4-diaminobutyric acid transaminase & $e c t B$ & EGP18460 & 46 & 5.69 \\
\hline & Ectoine synthase & ectC & EGP18459 & 15 & 5.03 \\
\hline & Ectoine hydroxylase & $e c t D$ & EGP18127 & 36 & 5.35 \\
\hline
\end{tabular}

a, GenBank accession number.

b, Molecular mass $(\mathrm{kDa})$ and pl were calculated through ExPASy Proteomics server (http://web.expasy.org/compute_pi/).

percentages of genome coverage were $102.64 \%$ and 99.56\% based on k-mer analysis and reads comparison, respectively. 3,894 Open Reading Frames (ORF) predicted by Glimmer had occupied $89.18 \%$ of the whole assembled sequences. The GC content within coding sequences was $53.23 \%$, which was a little higher than that of the whole sequences (52.57\%). Most putative proteins were distributed within the length ranges between 200 and 300 amino acids (aa) (Additional file 2, Figure S1). In addition, the putative proteins were classified according to the COG function (Additional file 3, Figure S2A) and KEGG pathway (Additional file 3, Figure S2B). The comparison of the predicted protein sequence set of Halomonas sp. TD01 with the nr database (NCBI) revealed its close relationship with Halomonas elongata DSM 2581, Chromohalobacter salexigens DSM 3043 and Aromatoleum aromaticum EbN1. Although the sequence of the scaffolds of Halomonas sp. TD01 have not been confirmed, the alignment between the chromosome of $C$. salexigens and the scaffolds of Halomonas sp. TD01 showed distinguishable co-linearity (Additional file 4, Figure S3).

\section{Identification and evolutionary analysis of PHA relevant genes from Halomonas sp. TD01}

The genes relevant to PHA were identified through homologous alignments against the public annotation databases, including KEGG, COG, Swiss-Prot, TrEMBL and $\mathrm{nr}$, using the BLAST program. As inferred from the similarity results, there might be two putative genes encoding PHA synthases (PhaC), three encoding PHA depolymerases (PhaZ), one encoding phasin (PhaP) and one encoding PHA synthesis repressor/regulator (PhaR, which showed 45\% identities with YP_725943 of Ralstonia eutropha $\mathrm{H16}$ ) in the genome of halophilic PHBV producing strain Halomonas sp. TD01 (Table 1). In addition, although the putative PhaC1 shared only $19 \%$ identity with PhaC2, both of them hit COG (clusters of orthologous groups) 3243 in the conserved domain database (CDD) at NCBI, implying that they belonged to PHA synthases [35]. Similarly, all the three PhaZs deduced from the genome of Halomonas sp. TD01 shared remarkably the same hits on COG3509 and pfam10503 in CDD, which strongly proposed their functions as PHB depolymerases. Phylogenetic trees clearly illustrated that the putative PhaZ, PhaP and PhaR from Halomonas sp. TD01 had close relationships with the corresponding, well-characterized enzymes from nonhalophiles, which presented clear, in silico evidence for their function in PHA degradation and regulation (not shown).

The evolutionary differences of PHA synthases between archaeal-, bacterial- and non-halophiles were revealed by phylogenetic analysis (Figure 1). PHA synthases are clustered into different groups (Figure 1). The PhaCs from haloarchaea shared general features with class III enzymes, forming a novel subclass; the PhaCs from halophilic bacteria were closer to class I enzymes [15]. PHA synthases of class I and II appeared to originate from a common ancestor, while synthases of class III and IV originated from an alternative common ancestor (Figure 1). However, the common ancestor of putative $\mathrm{PhaC} 2$ from strain TD01 and its homologues seemed relatively distant from the well-characterized PHA synthases in the phylogenetic analysis (Figure 1). Furthermore, the molecular masses of putative $\mathrm{PhaC} 2$ and its homologues were between 81 and $92 \mathrm{kDa}$, while the Class I enzymes are usually between 61 and $73 \mathrm{kDa}$ [1]. Revealed by the multiple alignments, the putative $\mathrm{PhaC} 2$ from Halomonas sp. TD01 shared longer C-terminus with those homologues from H. elongata DSM 2581, C. salexigens DSM 3043 and $A$. aromaticum EbN1 than that of the well-studied classical class I PHA synthases (Figure 2). It is possible that the longer $\mathrm{C}$-terminus of the $\mathrm{PhaC} 2$ was important 


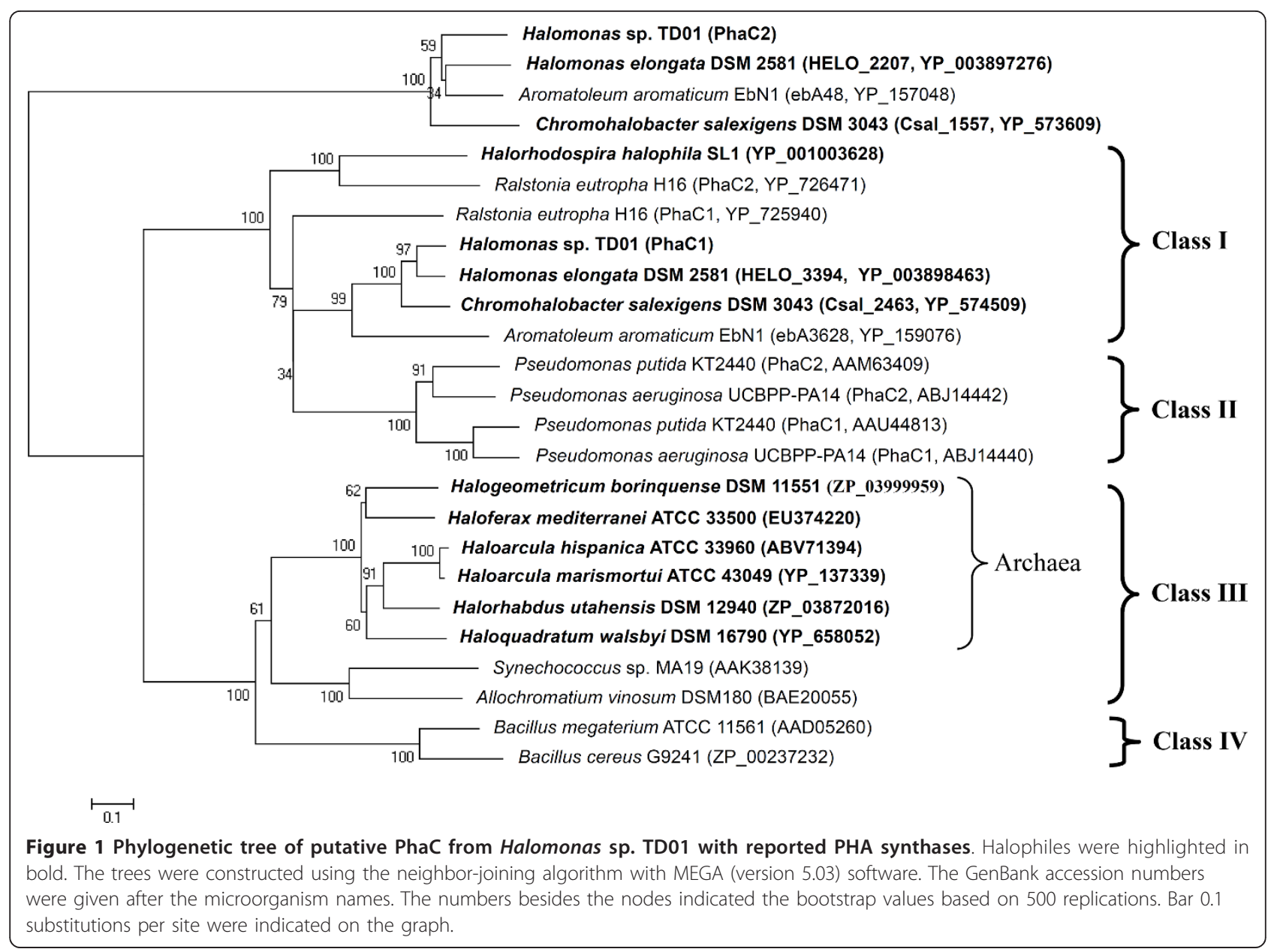

for the function of PHA synthase at high salt concentration, which was observed in the haloarchaeal PhaC $[15,16]$. However, their $\mathrm{N}$-terminus, which proved quite important for the function of classical PHA synthases $[36,37]$, was shorter than that of the well-studied classical class I PHA synthases (Figure 2). Yet, enzymatic and structural studies are required to determine whether the putative PhaC2 from Halomonas sp. TD01 and its homologues are a novel subclass of PHA synthases. Although there is still uncertainty about the classification of $\mathrm{PhaC} 2$ from Halomonas sp. TD01, PhaC1 from the strain TD01 exhibited relatively high similarities with the classical class I PHA synthases. For example, PhaC1 from TD01 has high sequence similarity (44\% and $34 \%$ sequence identities with YP_725940 and YP_726471, respectively) with the known class I PHA synthases from Ralstonia eutropha H16 (Figure 1). The molecular mass of PhaC1 was about $70 \mathrm{kDa}$, which is within the common range of well-studied class I enzymes [1]. In addition, the conserved lipase-like box residues were recognized in the PhaC1 enzyme (Figure 2).
The detailed analysis through multiple sequences alignments of putative $\mathrm{PhaC} 1$ and $\mathrm{PhaC} 2$ with their homologues was performed. The most similar homologues of both $\mathrm{PhaC} 1$ and $\mathrm{PhaC} 2$ came from $H$. elongata DSM 2581 (78\%, 73\% identities, respectively), C. salexigens DSM 3043 (67\%, 67\% identities, respectively) and A. aromaticum $\mathrm{EbN} 1$ (48\%, 69\% identities, respectively) (Additional file 5, Table S2). The multiple sequences alignments showed strong conservation between these protein sequences and the well-characterized PHA synthases (Figure 2). The conserved lipase-like box residues were recognized in all of these proteins (Figure 2). In addition, three residues (cysteine, aspartic acid and histidine), composing the conserved catalytic triad of PHA synthases, were also identified in these proteins (Figure 2). However, it is interesting to find that, instead of the traditional lipase-box pattern "GxCxG", all PhaC1 homologues (listed in Figure 2, except for YP_159076 from non-halophilic $A$. aromaticum EbN1), have "SxCxG", and all the PhaC2 homologues (listed in Figure 2) have "GxCxA". These interesting patterns may 


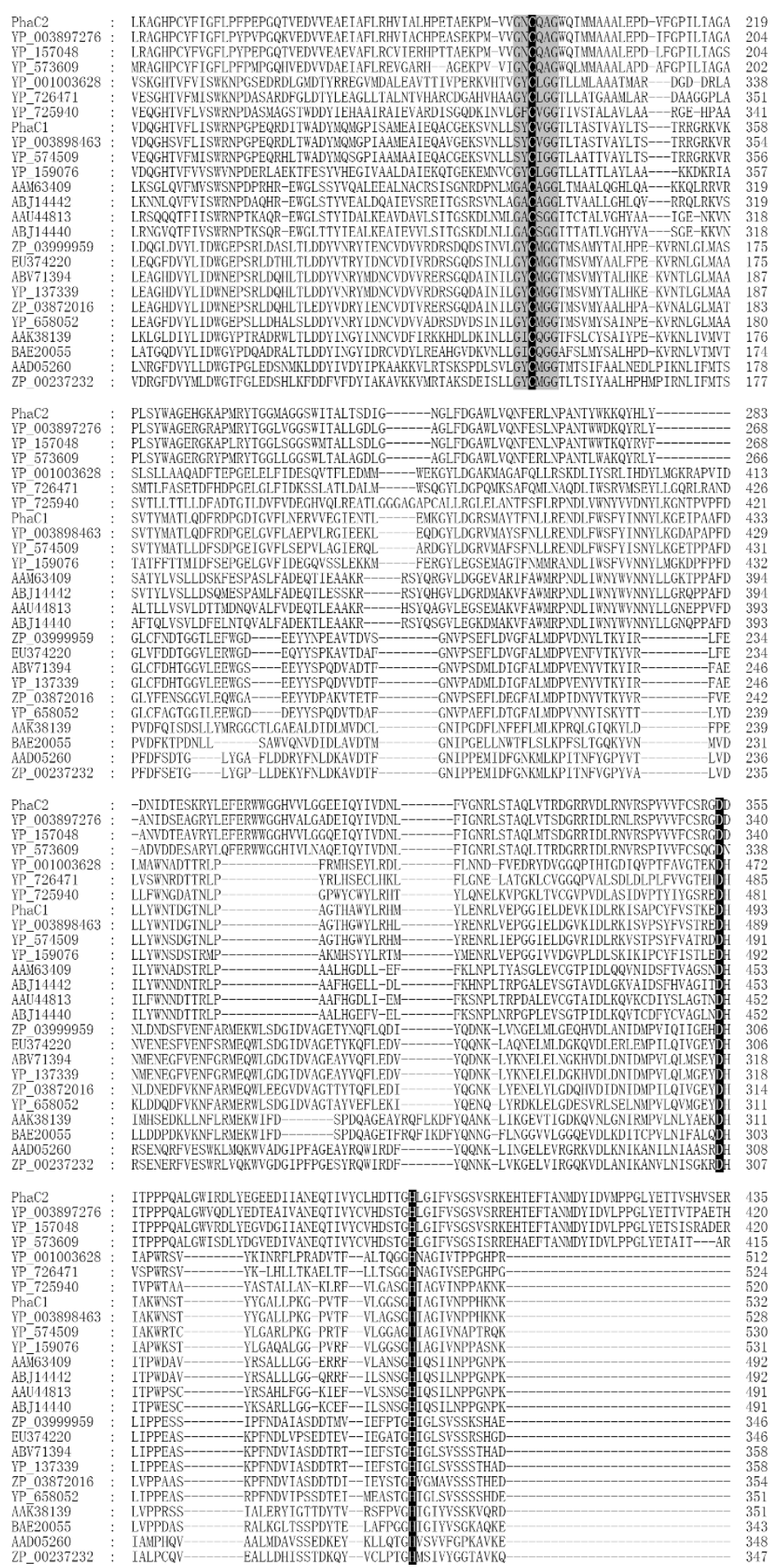

Figure 2 Multiple alignment of putative PhaC from Halomonas sp. TD01 with reported PHA synthases. Black shading indicated the conserved catalytic triad residues, and gray shading indicated lipase-like box residues. The sequences were listed in the same order of the phylogenetic tree in Figure 1. 
imply the existence of a new class of PHA polymerases among the halophilic bacteria. Yet, more experimental data are needed to elucidate whether these patterns are related to the hypersaline environments.

With the progress on the research of PhaCs from halophiles, our vision on PHA synthesis in these strains was amazingly broadened [38]. In contrast, few efforts had been devoted to the studies on other enzymes involved in PHA metabolism excluding PhaC. The putative PhaZ3 was supposed to be a secreted protein through the prediction of signal peptides (Additional file 6, Figure S4), whereas the other two PHA depolymerases (PhaZ1 and PhaZ2) lack signal peptides and may be responsible for the intracellular PHA degradation.

Most of PHA relevant genes are scattered in the genome, except for the phaP and phaC1, which were connected with a space of 92 bp (Figure 3A). However, an independent promoter of $p h a C 1$, with highly conserved -35 and -10 binding sites for $\sigma^{70}$, was identified (Figure 3B). The -35 motif extended into the tail of phaP coding region, and the putative transcription start site (TSS) was $73 \mathrm{bp}$ on the upstream of the translation start site (TLS) of phaC1 gene (Figure 3B). The existence of an independent promoter of phaC1 suggested the separated regulation on the gene expression of phaP and phaC1. It was understandable that phasins were usually expressed in large quantities during PHA storage period, whereas massive expression of PHA synthases was required during synthesis period $[39,40]$.

\section{Osmoregulatory mechanisms of Halomonas sp. TD01 inferred from the genome information}

The $\mathrm{pI}$ values provide simple indication of protein acidity. To compare the differences of protein acidity between halophiles and non-halophiles, the $\mathrm{pI}$ distribution of representative proteome from halophilic bacterium Halomonas sp. TD01 and Halomonas elongata DSM 2581, halophilic arhaeon Haloarcula marismortui ATCC 43049 and non-halophilic E. coli MG1655, was calculated with the interval of $0.2 \mathrm{pI}$ from 3 to 13. Bimodal distribution of $\mathrm{pI}$ appears clearly on the plot for each strain (Additional file 7, Figure S5). Both acidic peaks of Halomonas sp. TD01 and H. elongata DSM 2581 were at 5.1, which were a little lower than the non-halophilic E. coli MG1655 (at 5.5), but much higher than the extremely halophilic archaeon $H$. marismortui (at 4.3). While H. marismortui only showed a small peak representing basic proteins, the other three strains shared the distinct basic peaks at around pI 9.5. The median $\mathrm{pI}$ values of Halomonas sp. TD01, H. elongata, H. marismortui and E. coli were 5.67, 5.51, 4.46 and 6.17 , respectively, which displayed a similar pattern with the pI distribution.

As moderate halophiles, accumulation of organic osmolytes, instead of inorganic ions was supposed to be the main strategy to deal with high osmotic environment [8]. So far, many organic solutes, characterized with small molecule masses and fast turnover through de novo synthesis or absorption from environment and degradation, were identified to function as osmoregulators [41]. The genes relevant to betaine and ectoine, two of the most widespread compatible solutes in nature, were identified and annotated based on the genome information of Halomonas sp. TD01. There were two putative genes clusters encoding compatible solute synthesis systems for betaine (bet $A B I$ ) and ectoine $($ ect $A B C)$ (Table 1 and Additional file 8, Table S3). The genes $($ doe $A B C D)$ involved in the degradation of compatible solutes were also discovered in the genome of Halomonas sp. TD01 (Additional file 8, Table S3) [41]. In addition, five genes encoding the ATP-binding cassette $(\mathrm{ABC})$-type proline/glycine betaine transporters (ProU) and eight genes encoding betaine/carnitine/

A.

(552 bp)

(1851 bp)

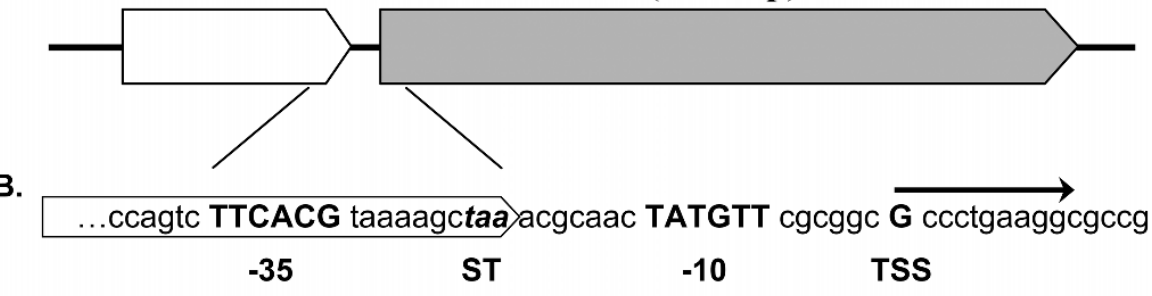

cgttctttggatgatgtgcctgggcagataatgccaagcaggtgagggagagtgagt Atgctg ...

Figure 3 The organization of phaP and phaC1. (A) The phasin gene (phaP) was located on the upstream of phaCl with a space of 92 bp. (B) The predicted promoter structure of phaC1. Bold capital letters indicated key base pairs. TSS, transcription start site; TLS, translation start site of phaC1; ST, stop codon of phaP; -10, Pribnow box; -35, -35 element. Arrow indicated the transcription from TSS. 
choline transporters (BCCT family) were identified through the comparison with well-studied betaine transporters (Additional file 8, Table S3) [42]. The ABC-type transporters generally showed broader substrate specificities than the ones belonging to the BCCT family [43]. In contrast, only one gene (tea $A$ ) encoding a homologue of tripartite ATP-independent periplasmic transporters (TRAP-T) for ectoine was unveiled (Additional file 8, Table S3) [44]. Most of these osmolytes relevant genes shared close similarities with the homologues from $C$. salexigens and $H$. elongata, as revealed by evolutionary analysis (Additional file 5, Table S2). These similarities were also observed in their PHA relevant genes (Additional file 9, Figure S6) and 16S rDNA sequences (Figure 4) based phylogenetic analysis.

\section{The evolutionary analysis of PHA and ectoine relevant proteins from Halomonas sp. TD01}

As revealed through the analysis of the osmoregulatory mechanism, Halomonas sp. TD01 was possibly able to synthesize ectoines with commercial interest as protectants against proteolysis [18]. The existence of PHA and ectoine synthesis genes qualifies Halomonas sp. TD01 as a candidate for the combined production of PHA and osmolytes [7]. Moreover, it was interesting to observe that many species other than halophiles possessed both homologues of putative PHA and ectoine relevant enzymes from Halomonas sp. TD01 (Additional file 1, Table S1). Based on the evolutionary distances of $16 \mathrm{~S}$ rDNA (Figure 4), PHA and ectoine relevant protein sequences, Halomonas sp. TD01 shared closest relationship with Chromohalobacter salexigens DSM 3043 and Halomonas elongata DSM 2581, which were also known as halophilic bacteria and belonged to the same family Halomonadaceae as the strain TD01 (Additional file 5, Table S2). It was interesting to find that, with regard to PHA relevant enzymes, similarities (the average evolutionary distance of PhaCs: 0.666 ) between TD01 and rhizobia strains, which fix nitrogen and are symbiotic with plant roots, were significant (Additional file 5, Table S2). The homologues of ectoine synthesis and PHA degradation (PhaZs) enzymes from Halomonas sp. TD01 were present in some species belonging to the class Actinobacteria, are a group of Gram-positive bacteria with high $\mathrm{G}+\mathrm{C}$ content (Additional file 5, Table S2). In addition, it was noticed that only proteins from some $\gamma$-proteobacteria species exhibited significant similarity with the PhaP from strain TD01 (Additional file 5, Table S2), which implies this PhaP possibly possesses a relative independent evolution pathway in the $\gamma$-proteobacteria. However, it seemed that the other homologues of strain TD01 spread in $\alpha-, \beta-, \gamma$-proteobacteria (Additional file 5, Table S2), suggesting that PHA and ectoine related enzymes from the halophilic strain TD01 shared common ancestors with the non-halophiles.
The plots of evolutionary distances of PHA and ectoine relevant enzymes versus that of $16 \mathrm{~S}$ rDNA were drawn with the aim of better understanding of the evolutionary relationship among these genes and $16 \mathrm{~S}$ rDNA (Figure 5). When the evolutionary distances of $16 \mathrm{~S}$ rDNA were lower than 0.15 , there was an obvious colinearity between the evolutionary distances of $16 \mathrm{~S}$ rDNA and those of the ectoine relevant enzymes (Figure $5 \mathrm{~B})$. Yet, among the PHA relevant enzymes, only the evolutionary distances of $\mathrm{PhaC} 1$ and PhaP showed some co-linearity with those of $16 \mathrm{~S}$ rDNA when the evolutionary distances of $16 \mathrm{~S}$ rDNA were lower than 0.15 (Figure 5A). However, when the evolutionary distances of $16 \mathrm{~S}$ rDNA were higher than 0.15 , the evolutionary distances of PHA and ectoine relevant enzymes seemed non-colinear with those of $16 \mathrm{~S}$ rDNA (Figure 5). Inferred from this non-colinearity, there may be extensive horizontal gene transfer of PHA and ectoine relevant genes between the ancestors of these species listed in Additional file 1, Table S1. For example, although Azotobacter vinelandii DJ was evolutionarily distant from strain TD01, their $p h a C$ and phaR genes shared significant similarities; the phaR genes of Achromobacter piechaudii ATCC 43553, which was distantly related to TD01 with respect to $16 \mathrm{~S}$ rDNA sequences, shared significant similarities with that of TD01 (Additional file 5, Table S2). Although PhaC, PhaP, PhaR, and PhaZ genes were all involved in PHA metabolisms, they were under different horizontal gene transfer (Figure 5A). The fact that there were similar evolutionary distances of ectoine genes from closely or distantly related genomes to Halomonas sp. TD01 (Figure 5B), may suggest that there were also horizontal gene transfer of ectoine genes. Mycobacterium smegmatis str. MC2 155 and Stackebrandtia nassauensis DSM 44728, for instance, were distantly related to strain TD01 based on 16S rDNA sequences, they shared similar ectoine genes with that of Halomonas sp. TD01 (Additional file 5, Table S2). Moreover, there were some similarities between the phylogenetic trees of 16S rDNA (Figure 4) and PHA, ectoine relevant enzymes (Additional file 9, Figure S6), especially for the closely related strains Halomonas elongata DSM 2581, Chromohalobacter salexigens DSM 3043. Although the sequence similarity of PHA and ectoine relevant genes implies the existence of horizontal gene transfer events between different species, no distinguishable transposable elements, which were identified through the RepeatMasker and RepeatProteinMasker [28], were found flanking these genes in the genome of Halomonas sp. TD01 (Additional file 10, Table S4 and S5). There remained a challenge to disclose how these genes transferred between the different species.

To further understand the functional relationships between PHA and ectoine relevant enzymes, Pearson 


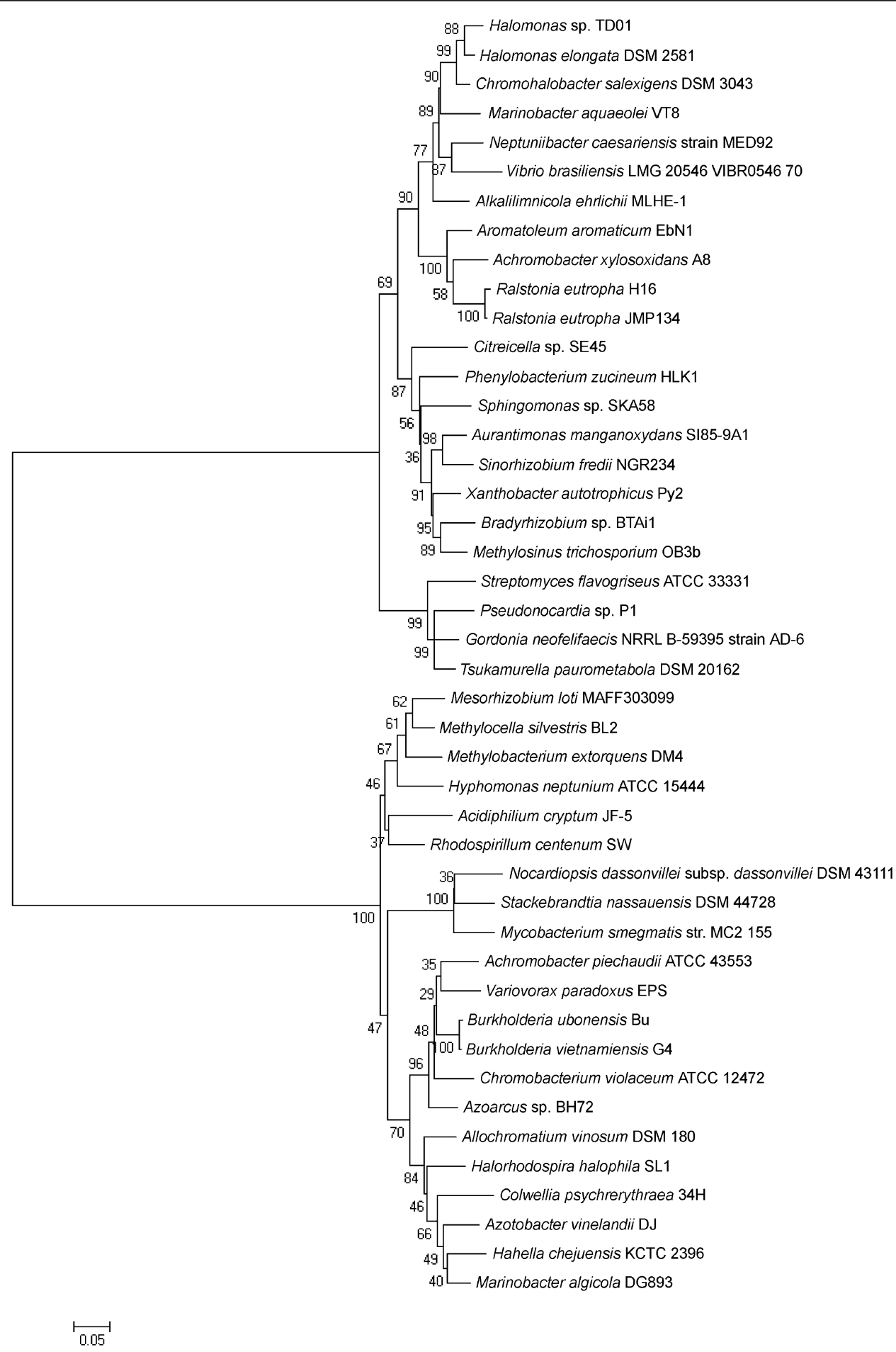

Figure 4 Phylogenetic tree based on the 16S rDNA sequences of strains with homologues of PHA and ectoine relevant enzymes. The trees were constructed using the neighbor-joining algorithm with MEGA (version 5.03) software. The numbers besides the nodes indicated the bootstrap values based on 500 replications. Bar 0.05 substitutions per site were indicated on the graph. The GenBank accession numbers of 165 rDNA sequences of the strains with the homologues of PHA and ectoine relevant enzymes were listed in Additional file 1, Table S1. 


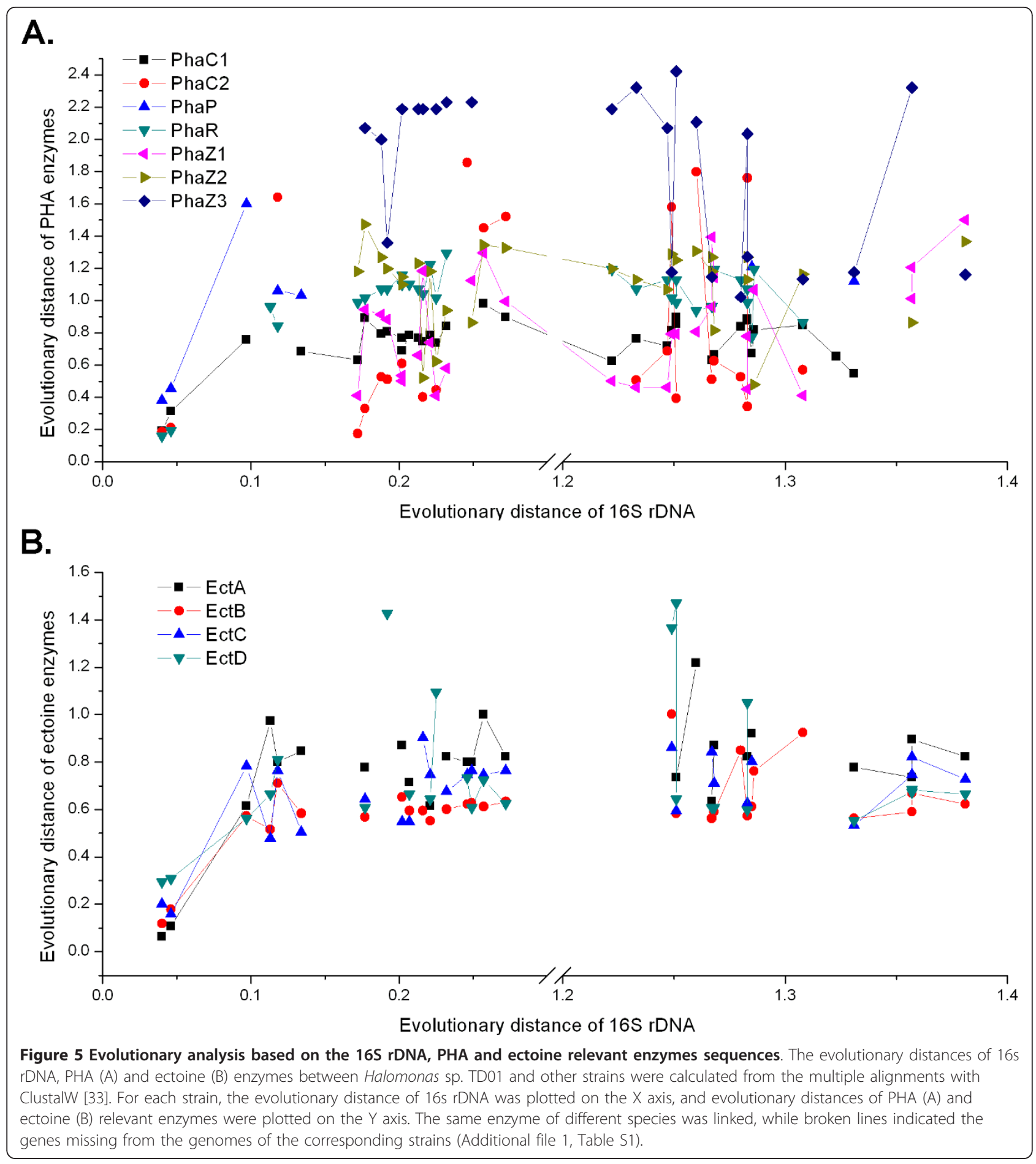

correlation coefficient was calculated after the transformation of evolutionary distances, with the aim of illustrating the evolutionary pressure imposed to keep any two genes in the genome. It was obvious that EctA, $E c t B, E c t C$, and EctD were highly related to each other, as they almost co-occur in the genome of all strains investigated in this study, and the correlations of their evolutionary distances to those in TD01 were very high (Figure 6). This was in agreement with the fact that they formed an operon in the genome of some strains, such as Sphingomonas sp. SKA58 and Stackebrandtia nassauensis DSM 44728, etc (Additional file 1, Table S1). In addition, as elucidated in the heatmap and cluster dendrogram, PhaZ1, PhaZ2 and PhaZ3 were closely 


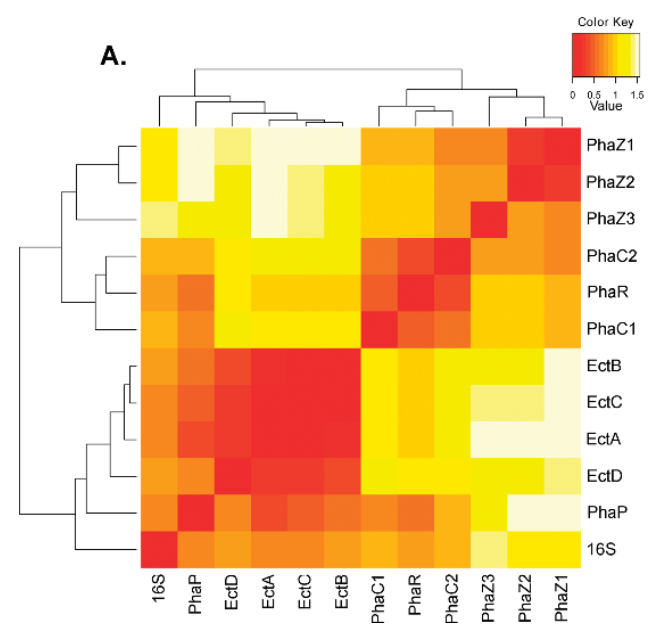

B.

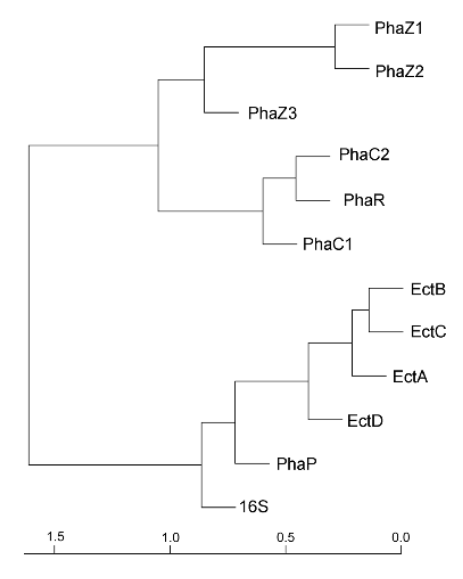

Figure 6 The correlation of evolutionary distance for any two of the 16S rDNA, PHA and ectoine relevant sequences. The heatmap (A) and cluster dendrogram (B) based on their evolutionary correlation coefficient. The self-distance was set at zero. Then, the heatmap was plotted by using the function heatmap. 2 in package gplots of R programming language. The color intensity in the heatmap corresponded to the distance. The cluster dendrogram was plotted by the hierarchical clustering method in $\mathrm{R}$ programming language.

related to each other, and all of them share distinct relationship with other PHA genes (Figure 6). Within these three PHA depolymerases, PhaZ1 and PhaZ2 were more closely related to each other than to PhaZ3, which was in accordance to the fact that PhaZ1 and PhaZ2 were putative intracellular depolymerases, whereas PhaZ3 was putative extracellular depolymerase, as suggested by the signal peptide prediction (Additional file 6, Figure S4). Although PhaP seemed evolutionarily closer to ectoine relevant enzymes, this was probably because it was absent in 36 out of 44 strains (Additional file 1, Table $\mathrm{S} 1$ ). More studies including both in silico and wetexperiment, should be implemented for further clarification of their relationships. As illustrated by Figure 6, it seemed that there were not high evolutionary pressures to keep PHA genes together in the same genome, and whether to keep or lose certain PHA genes may be dependent on specific environment of each strain. For instance, Ralstonia eutropha H16, a ubiquitous inhabitant of soil and freshwater, suffers most from energy limitation, and has been well adapted with the accumulation of high content PHA as carbon reservoir. Therefore, multiple PHA relevant genes were maintained in its genome, whereas ectoine genes were missing as there was no environmental pressure for the presence of ectoines. This suggested that genetic engineering of single PHA related genes, such as knockout or addition of PHA genes, may not have astonishing impacts on bacterial growth and PHA production, yet it may be necessary in order to achieve higher PHA production in some strains, such as Halomonas sp. TD01. In contrast, the evolutionary pressures to keep ectoine genes together in the same genome were much higher (Figure 6), suggesting these genes may all be necessary for ectoine metabolism. Thus, inhibiting the expression of any of the four ectoine genes may have a strong impact on the ectoine biosynthesis and the survival in high osmotic environments. This finding may help develop better genetic engineering strategies for future production of PHA and ectoine.

\section{Discussion}

So far, there have been relatively few studies on the PHA synthases from halophilic microorganisms [7]. Recently, the detailed studies on the PHA synthases from the halophilic archaea have extended our insights on their classification, providing solid evidences to support the existence of a novel subclass (IIIA) synthases with distinguished features from the subclass (IIIB) isolated from bacteria [15]. The widespread novel PHA synthases in halophilic archaea consist of two subunits, $\mathrm{PhaC}$ and PhaE, both of which are required for their activities [38]. However, these novel PHA enzymes are clustered separately from the ones isolated from the halophilic bacteria, including Halomonas sp. TD01, $H$. elongata, C. salexigens, and Halorhodospira halophila, etc (Figure 1). As illustrated by the phylogenetic analysis, PHA synthases (especially the putative PhaC1) from halophilic bacteria, shared the significant similarities with class I enzymes, which were different from the well-studied enzymes from halophilic archaea (Figure 1) [7]. PhaCs in Halomonas sp.TD01 were able to synthesize PHB with a molecular weight of $600 \mathrm{kDa}$ in fedbatch fermentation, which was in accordance with the characteristics of class I PHA synthases [1,13]. The conserved catalytic residues, firstly discovered in the PHA 
synthases of Chromatium vinosum and $R$. eutropha, were also recognized in PhaC1 and PhaC2 from Halomonas sp. TD01 (Figure 2), indicating that they share similar catalytic mechanisms and substrate specificities $[7,45]$. In addition, PHBV was detected when glucose and propionic acid (or valeric acid) were supplemented as carbon sources in the medium, which also agreed with the features of class I synthases [13]. Under the catalysis of putative PhaC1 and/or PhaC2, PHBV accumulation was able to reach $80 \mathrm{wt} \%$ of cell dry weight consisting of 30\% 3-hydroxyvalerate 3HV fraction when supplemented with valeric acid as additional carbon source, which qualified Halomonas sp. TD01 as a strain of industrial interest for PHBV production [13]. Nevertheless, physiological and enzymatic characterization of the PHA synthases from halophilic bacteria are still required to elucidate whether the putative $\mathrm{PhaC} 2$ from Halomonas sp. TD01 and its homologues form their own class of PHA synthases, as implied by the phylogenetic studies and multiple sequences alignment of putative PhaC2 (Figure 2).

While PHA synthases from halophiles have received attentions, the studies on PHA depolymerase, regulator/ repressor and phasin from halophiles, which also play crucial roles in the PHA cycle, remained unclear [15]. Evolutionary analysis demonstrated that the PhaC, PhaZ, PhaP, PhaR and ectoine relevant enzymes from halophilic Halomonas sp. TD01 share close relationships (the average evolutionary distance: 0.945 ) with those from non-halophilic strains (Additional file 5, Table S2). It is very clear that further systematic and detailed studies on these genes from halophiles would improve our understanding on their functions and evolution, potentially leading to enhancing PHA production at reduced costs. Moreover, genome-scale metabolic analysis would also advance the progress of PHA production by halophiles [46].

The most well-known genetic organization of representative PHA synthases in class I was the phbCAB operon from $R$. eutropha, containing genes encoding PHA synthases, acetyl-CoA acetyltransferase and 3hydroxybutyryl-CoA dehydrogenase in sequence [1]. However, in Halomonas sp. TD01, phaC and phaA, phaB genes were scattered throughout the genome (Table 1, Additional file 8, Table S3). More interestingly, the neighborhood genetic organization of the homologues of putative phaP and phaC1 from Halomonas sp. TD01 was also conserved in both halophiles and nonhalophiles exclusively from $\gamma$-proteobacteria, which can imply that there may be extensive horizontal gene transfers between their ancestral strains, possibly suggesting a same living environment in the past (Table 2). The interval lengths between phaC1 and phaP genes varied from 55 to 204 bp (Table 2). Most of these species were isolated from an aquatic (especially oceanic) environment, similar to the saline lake from where Halomonas sp. TD01 was isolated [13]. It is possible that the horizontal gene transfer may have occurred in ancient times, when the universal common ancestors of these $\gamma$ proteobacteria mentioned in Table 2 survived in the same aquatic environment, leading to the acquisition of adjacent phaP and phaC1 genes by the ancestor of Halomonas sp. TD01. With the geological evolution, the ancestors of these $\gamma$-proteobacteria were then separated into their present environments and the phaCl and phaP genes with their interval sequences evolved independently to their current status. The conservation of adjacent organization could play an important role in the regulation and metabolism of PHA cycles in $\gamma$-proteobacteria, which deserves further study.

Wild-type cells require PHA synthesis and degradation cycle to maintain carbon supply balance under changing environments [2]. However, for industrial production of PHA, degradation is not favored, because it leads to the waste of carbon sources and consequently reduces PHA accumulation $[47,48]$. For example, it has been reported that the knockout of phaZ in P. putida KT2442 inhibited the decrease of PHA content during batch fermentation processes [47]. For Halomonas sp. TD01, the presence of three putative PHA depolymerases likely decreased PHA accumulation in batch fermentation processes (unpublished observations). Thus, identification of these phaZ genes could help the intentional knockout of PHA degradation pathways in Halomonas sp TD01, in the hope of promoting more PHA accumulation. In addition, distinguishing the intracellular (PhaZ1 and PhaZ2) and extracellular (PhaZ3) depolymerases allow the possibility to identify targets of most importance, reducing the time and labor required for gene knockout.

To maintain the balance of osmotic pressure across the cell membrane, high concentrations of ions or organic osmolytes are accumulated in the cells [49]. Neutral proteins would become denatured in hypersaline condition, while acidic ones, with more negatively charged amino acid residues on the surfaces, were able to stay functional through the binding of hydrated salt ions in the cytoplasm [50]. It was reported that the proteomes of extremely halophilic archaea, such as $H$. marismortui, showed a notable general tendency towards acidity, which was illustrated by the pI distribution (Additional file 7, Figure S5) [49]. Contrary to this, less acidic proteomes from halophilic bacteria, including Halomonas sp. TD01 and H. elongata, were observed and implied their decreased dependence on the absorption of salt to maintain ionic balance in hypersaline environments [51]. To survive in hypersaline conditions, it was believed that halophilic Halomonas sp. TD01 adopted another universal strategy, accumulating 
Table 2 The intervals lengths between the co-linear phaP and phaC1 genes from Halomonas sp. TD01 and their homologues in other microorganisms

\begin{tabular}{|c|c|c|c|}
\hline Order & Family & Species & Interval (bp) \\
\hline \multirow[t]{5}{*}{ Oceanospirillales } & Halomonadaceae & Halomonas sp.TD01 & 92 \\
\hline & & Halomonas elongata DSM 2581 & 82 \\
\hline & & Chromohalobacter salexigens DSM 3043 & 204 \\
\hline & Oceanospirillaceae & Marinomonas sp. MED121 & 92 \\
\hline & Hahellaceae & Hahella chejuensis KCTC 2396 & 134 \\
\hline \multirow[t]{25}{*}{ Vibrionales } & Vibrionaceae & Vibrio alginolyticus 12G01 & 66 \\
\hline & & Vibrio brasiliensis LMG 20546 & 55 \\
\hline & & Vibrio caribbenthicus ATCC BAA-2122 & 57 \\
\hline & & Vibrio cholerae 1587 & 57 \\
\hline & & Vibrio coralliilyticus ATCC BAA-450 & 58 \\
\hline & & Vibrio furnissii CIP 102972 & 56 \\
\hline & & Vibrio harveyi 1DA3 & 66 \\
\hline & & Vibrio metschnikovii CIP 69.14 & 116 \\
\hline & & Vibrio mimicus VM573 & 57 \\
\hline & & Vibrio orientalis CIP 102891 & 57 \\
\hline & & Vibrio parahaemolyticus RIMD 2210633 & 66 \\
\hline & & Vibrio shilonii AK1 & 79 \\
\hline & & Vibrio sinaloensis DSM 21326 & 58 \\
\hline & & Vibrio sp. Ex25 & 80 \\
\hline & & Vibrio sp. RC586 & 57 \\
\hline & & Vibrio splendidus LGP32 & 73 \\
\hline & & Vibrio vulnificus CMCP6 & 61 \\
\hline & & Vibrionales bacterium SWAT-3 & 75 \\
\hline & & Grimontia hollisae CIP 101886 & 63 \\
\hline & & Photobacterium angustum S14 & 211 \\
\hline & & Photobacterium damselae subsp. damselae CIP 102761 & 59 \\
\hline & & Photobacterium leiognathi subsp. mandapamensis svers.1.1. & 119 \\
\hline & & Photobacterium profundum 3TCK & 179 \\
\hline & & Photobacterium profundum SS9 & 65 \\
\hline & & Photobacterium sp. SKA34 & 121 \\
\hline Pseudomonadales & Moraxellaceae & Enhydrobacter aerosaccus SK60 & 199 \\
\hline \multirow[t]{4}{*}{ Alteromonadales } & Shewanellaceae & Shewanella halifaxensis HAW-EB4 & 94 \\
\hline & & Shewanella pealeana ATCC 700345 & 95 \\
\hline & Alteromonadaceae & Marinobacter aquaeolei VT8 & 74 \\
\hline & & Marinobacter sp. ELB17 & 118 \\
\hline \multirow[t]{4}{*}{ Aeromonadales } & Aeromonadaceae & Aeromonas caviae & 92 \\
\hline & & Aeromonas hydrophila & 92 \\
\hline & & Aeromonas hydrophila subsp. hydrophila ATCC 7966 & 93 \\
\hline & & Aeromonas salmonicida subsp. salmonicida A449 & 93 \\
\hline
\end{tabular}

organic compatible solutes instead of inorganic ions to balance the osmotic pressure with a surprisingly broad salt concentration range [8]. This was illustrated by the widespread of biosynthesis and transporter genes for multiple organic osmolytes over the genome. Even though halophilic microorganisms seemed to optimize their metabolism to minimize the energetic cost for osmotic adaptation, the de novo biosynthesis of osmolytes was less favorable with respect to either energy consumption or flexibility than their absorption from environment [52]. In addition, there are many transporters for betaine, which possibly suggested that betaine was also one of the compatible solutes utilized by Halomonas sp. TD01 [53]. However, a complete comprehension of the occurrence and distribution of organic osmoregulatory solutes in Halomonas sp. TD01 remain to be determined. Moreover, as the environmental factors, especially the nutritional ones, are able to strongly influence the production of PHA, the effects of both environmental factors and genetic background of 
Halomonas sp. TD01 on the production of PHA should be further studied.

\section{Conclusions}

In conclusion, the disclosure of the genome sequences of Halomonas sp. TD01 improves our understanding on the metabolism and evolutionary relationship of PHA and osmoregulatory solutes from halophilic bacteria. Detailed and systematic in silico analysis on the PHA and osmolytes relevant genes provides abundant insights on their classifications, functions and phylogeny. Osmoregulatory mechanisms were also discussed through the comparison of pI distribution. The availability of genomic information would inevitably pave new ways for the application of numerous post-genomic technologies and accelerate our work in the genetic optimization of Halomonas sp. TD01 for the industrial production of $\mathrm{PHA}$, and possibly accompanied with the co-production of compatible solute ectoine.

\section{Additional material}

Additional file 1: Table S1. Accession numbers of 165 rDNA, putative PHA and ectoine relevant proteins from Halomonas sp. TD01 and other strains.

Additional file 2: Figure S1. Gene length distribution of Halomonas sp. TD1.

Additional file 3: Figure S2. COG function and KEGG pathway classification of Halomonas sp. TD1.

Additional file 4: Figure S3. Alignment of the chromosomes of $C$. salexigens and Halomonas sp. TD1.

Additional file 5: Table S2. Evolutionary distances of 165 rDNA, PHA and ectoine relevant proteins between Halomonas sp. TD01 and other species.

Additional file 6: Figure S4. Signal peptide prediction of PhaZ3 with neural networks (NN) and hidden markov models (HMM).

Additional file 7: Figure S5. Calculated isoelectric point (pl) distribution. Isoelectric point (pl) distribution of halophilic bacteria (Halomonas sp. TD01 and Halomonas elongata DSM 2581), haloarchaea (Haloarcula marismortui ATCC 43049) and non-halophilic bacterium (Escherichia coli MG1655) versus percentage of total proteins. Distribution with the interval of $0.2 \mathrm{pl}$ was counted and plotted.

Additional file 8: Table S3. Accession numbers of putative PhaA, PhaB and osmolytes relevant enzymes in the genome of Halomonas sp. TD01.

Additional file 9: Figure S6. Phylogenetic trees based on the PhaC1 (A), PhaC2 (B), PhaP (C), PhaR (D), PhaZ1 (E), PhaZ2 (F), PhaZ3 (G), EctA (H), EctB (I), EctC $(J)$ and EctD $(K)$ sequences of Halomonas sp. TD01 with their homologues of other strains.

Additional file 10: Table S4 and S5. Locus of putative PHA and osmolytes relevant genes in the genome of Halomonas sp. TD01; Transposon and TEprotein identification through the RepeatMasker and RepeatProteinMasker.

\section{Acknowledgements}

We are grateful to lan Wimpenny from Keele University, for his help with the improvement of writing. This research was financially supported by 973 Basic Research Fund (Grant No. 2011CBA00807) and Natural Science Foundation of China (Grant No 31170099) as well as by National High Tech 863 Grant (Project No. 2010AA101607),

\section{Author details}

'Dept Biological Sciences and Biotechnology, MOE Key Lab. Bioinformatics (\& System Biology), Tsinghua University-Peking University Joint Center for Life Sciences, School of Life Sciences, Tsinghua University, Beijing 100084, China. ${ }^{2}$ School of Biological Sciences and Biotechnology, Xinjiang University, Urumchi, 830046, China. ${ }^{3}$ Institute of Biostatistics, School of Life Sciences, Fudan University, Shanghai, China.

\section{Authors' contributions}

$L C$ designed, performed most parts of the study, and prepared the manuscript. DT designed and performed sequencing of genome. GA and JCC participated in the screening of the strain and the sequencing of the genome. XRD performed the Pearson correlation study. WDT designed the evolutionary distance study and revised the manuscript. GQC designed and supervised the experiments, and revised the manuscript. All authors read and approved the final manuscript.

\section{Competing interests}

The authors declare that they have no competing interests.

Received: 8 August 2011 Accepted: 1 November 2011

Published: 1 November 2011

\section{References}

1. Rehm BHA: Polyester synthases: natural catalysts for plastics. Biochem $J$ 2003, 376:15-33.

2. Potter M, Steinbüchel A: Poly(3-hydroxybutyrate) granule-associated proteins: Impacts on poly(3-hydroxybutyrate) synthesis and degradation. Biomacromolecules 2005, 6:552-560.

3. Chen GQ: A microbial polyhydroxyalkanoates (PHA) based bio- and materials industry. Chem Soc Rev 2009, 38:2434-2446.

4. Joshi AA, Kanekar PP, Kelkar AS, Sarnaik SS, Shouche Y, Wani A: Moderately halophilic, alkalitolerant Halomonas campisalis MCM B-365 from Lonar Lake, India. J Basic Microbiol 2007, 47:213-221.

5. Budde CF, Riedel SL, Willis LB, Rha C, Sinskey AJ: Production of poly(3hydroxybutyrate-co-3-hydroxyhexanoate) from plant oil by engineered Ralstonia eutropha strains. Appl Environ Microbiol 2011, 77:2847-2854.

6. Park SJ, Choi Jl, Lee SY: Short-chain-length polyhydroxyalkanoates: Synthesis in metabolically engineered Escherichia coli and medical applications. J Microbiol Biotechnol 2005, 15:206-215.

7. Quillaguamán JH, Guzmán H, Van-Thuoc D, Hatti-Kaul R: Synthesis and production of polyhydroxyalkanoates by halophiles: current potential and future prospects. Appl Microbiol Biotechnol 2010, 85:1687-1696.

8. Oren A: Microbial life at high salt concentrations: phylogenetic and metabolic diversity. Saline Systems 2008, 4:2.

9. DasSarma P, DasSarma S: On the origin of prokaryotic "species": the taxonomy of halophilic Archaea. Saline Systems 2008, 4:5.

10. Kulkarni SO, Kanekar PP, Nilegaonkar SS, Sarnaik SS, Jog JP: Production and characterization of a biodegradable poly (hydroxybutyrate-cohydroxyvalerate) (PHB-co-PHV) copolymer by moderately haloalkalitolerant Halomonas campisalis MCM B-1027 isolated from Lonar Lake, India. Bioresour Technol 2010, 101:9765-9771.

11. Kulkarni SO, Kanekar PP, Jog JP, Patil PA, Nilegaonkar SS, Sarnaik SS, Kshirsagar PR: Characterisation of copolymer, poly (hydroxybutyrate-cohydroxyvalerate) (PHB-co-PHV) produced by Halomonas campisalis (MCM B-1027), its biodegradability and potential application. Bioresour Technol 2011, 102:6625-6628

12. Choi J, Lee SY: Factors affecting the economics of polyhydroxyalkanoate production by bacterial fermentation. Appl Microbiol Biotechnol 1999, 51:13-21.

13. Tan D, Xue YS, Aibaidula G, Chen GQ: Unsterile and continuous production of polyhydroxybutyrate by Halomonas TD01. Biorescour Technol 2011, 102:8130-8136.

14. Hezayen FF, Steinbüchel A, Rehm BHA: Biochemical and enzymological properties of the polyhydroxybutyrate synthase from the extremely halophilic archaeon strain 56. Arch Biochem Biophys 2002, 403:284-291.

15. Han J, Hou J, Liu HL, Cai SF, Feng B, Zhou JA, Xiang H: Wide distribution among halophilic archaea of a novel polyhydroxyalkanoate synthase 
subtype with homology to bacterial type III synthases. Appl Environ Microbiol 2010, 76:7811-7819.

16. Lu QH, Han J, Zhou LG, Zhou J, Xiang H: Genetic and biochemical characterization of the poly(3-hydroxybutyrate-co-3-hydroxyvalerate) synthase in Haloferax mediterranei. J Bacteriol 2008, 190:4173-4180.

17. Roberts MF: Organic compatible solutes of halotolerant and halophilic microorganisms. Saline Systems 2005, 1:5.

18. Kolp S, Pietsch M, Galinski EA, Gütschow M: Compatible solutes as protectants for zymogens against proteolysis. Biochim Biophys Acta 2006, 1764:1234-1242.

19. Delcher AL, Bratke KA, Powers EC, Salzberg SL: Identifying bacterial genes and endosymbiont DNA with Glimmer. Bioinformatics 2007, 23:673-679.

20. Ogata H, Goto S, Sato K, Fujibuchi W, Bono H, Kanehisa M: KEGG: Kyoto Encyclopedia of Genes and Genomes. Nucleic Acids Res 1999, 27:29-34.

21. Tatusov RL, Fedorova ND, Jackson JD, Jacobs AR, Kiryutin B, Koonin EV, Krylov DM, Mazumder R, Mekhedov SL, Nikolskaya AN, Rao BS, Smirnov S, Sverdlov AV, Vasudevan S, Wolf YI, Yin JJ, Natale DA: The COG database: an updated version includes eukaryotes. BMC Bioinformatics 2003, 4:41

22. Schneider $M$, Lane $L$, Boutet $E$, Lieberherr D, Tognolli M, Bougueleret $L$, Bairoch A: The UniProtKB/Swiss-Prot knowledgebase and its Plant Proteome Annotation Program. J Proteomics 2009, 72:567-573.

23. Boeckmann B, Bairoch A, Apweiler R, Blatter MC, Estreicher A, Gasteiger E, Martin MJ, Michoud K, O'Donovan C, Phan I, Pilbout S, Schneider M: The SWISS-PROT protein knowledgebase and its supplement TrEMBL in 2003. Nucleic Acids Res 2003, 31:365-370.

24. Altschul SF, Madden TL, Schäffer AA, Zhang J, Zhang Z, Miller W, Lipman DJ: Gapped BLAST and PSI-BLAST: a new generation of protein database search programs. Nucleic Acids Res 1997, 25:3389-3402.

25. Schattner P, Brooks AN, Lowe TM: The tRNAscan-SE, snoscan and snoGPS web servers for the detection of tRNAs and snoRNAs. Nucleic Acids Res 2005, 33:W686-W689.

26. Lagesen K, Hallin P, Rødland EA, Stærffeldt HH, Rognes T, Ussery DW: RNAmmer: consistent and rapid annotation of ribosomal RNA genes. Nucleic Acids Res 2007, 35:3100-3108.

27. Gardner PP, Daub J, Tate J, Moore BL, Osuch IH, Griffiths-Jones S, Finn RD, Nawrocki EP, Kolbe DL, Eddy SR, Bateman A: Rfam: Wikipedia, clans and the "decimal" release. Nucleic Acids Res 2011, 39:D141-D145.

28. Tarailo-Graovac M, Chen N: Using RepeatMasker to identify repetitive elements in genomic sequences. Curr Protoc Bioinformatics 2009, 4:10.

29. Reese MG: Application of a time-delay neural network to promoter annotation in the Drosophila melanogaster genome. Comput Chem 2001, 26:51-56.

30. Gordon JJ, Towsey MW, Hogan JM, Mathews SA, Timms P: Improved prediction of bacterial transcription start sites. Bioinformatics 2006, 22:142-148.

31. Bendtsen JD, Nielsen $H$, von Heijne G, Brunak S: Improved prediction of signal peptides: SignalP 3.0. J Mol Biol 2004, 340:783-795.

32. Papadopoulos JS, Agarwala R: COBALT: constraint-based alignment tool for multiple protein sequences. Bioinformatics 2007, 23:1073-1079.

33. Chenna R, Sugawara H, Koike T, Lopez R, Gibson TJ, Higgins DG, Thompson JD: Multiple sequence alignment with the Clustal series of programs. Nucleic Acids Res 2003, 31:3497-3500.

34. Tamura K, Peterson D, Peterson N, Stecher G, Nei M, Kumar S: MEGA5: Molecular evolutionary genetics analysis using maximum likelihood, evolutionary distance, and maximum parsimony methods. Mol Biol Evol 2011, 28:2731-2739.

35. Sayers EW, Barrett T, Benson DA, Bolton E, Bryant SH, Canese K, Chetvernin V, Church DM, DiCuccio M, Federhen S, Feolo M, Fingerman IM, Geer LY, Helmberg W, Kapustin Y, Landsman D, Lipman DJ, Lu Z, Madden TL, Madej T, Maglott DR, Marchler-Bauer A, Miller V, Mizrachi I, Ostell J, Panchenko A, Phan L, Pruitt KD, Schuler GD, Sequeira E, Sherry ST, Shumway M, Sirotkin K, Slotta D, Souvorov A, Starchenko G, Tatusova TA, Wagner L, Wang Y, Wilbur WJ, Yaschenko E, Ye J: Database resources of the National Center for Biotechnology Information. Nucleic Acids Res 2010, 38:D5-D16.

36. Rehm BHA, Antonio RV, Spiekermann P, Amara AA, Steinbüchel A: Molecular characterization of the poly(3-hydroxybutyrate) (PHB) synthase from Ralstonia eutropha: in vitro evolution, site-specific mutagenesis and development of a PHB synthase protein model. Biochim Biophys Acta 2002, 1594:178-190.
37. Schubert $P$, Krüger N, Steinbüchel A: Molecular analysis of the Alcaligenes Eutrophus poly(3-Hydroxybutyrate) biosynthetic operon: Identification of the $\mathrm{N}$-terminus of poly(3-Hydroxybutyrate) synthase and identification of the promoter. J Bacteriol 1991, 173:168-175.

38. Han J, Lu Q, Zhou L, Zhou J, Xiang H: Molecular characterization of the phaEC $\mathrm{Hm}_{\mathrm{m}}$ genes, required for biosynthesis of poly(3-hydroxybutyrate) in the extremely halophilic archaeon Haloarcula marismortui. Appl Environ Microbiol 2007, 73:6058-6065.

39. Hanley SZ, Pappin DJC, Rahman D, White AJ, Elborough KM, Slabas AR: Reevaluation of the primary structure of Ralstonia eutropha phasin and implications for polyhydroxyalkanoic acid granule binding. FEBS Lett 1999, 447:99-105.

40. Wieczorek R, Pries A, Steinbüchel A, Mayer F: Analysis of a 24-kilodalton protein associated with the polyhydroxyalkanoic acid granules in Alcaligenes Eutrophus. J Bacteriol 1995, 177:2425-2435.

41. Schwibbert K, Marin-Sanguino A, Bagyan I, Heidrich G, Lentzen G, Seitz H, Rampp M, Schuster SC, Klenk HP, Pfeiffer F, Oesterhelt D, Kunte HJ: A blueprint of ectoine metabolism from the genome of the industrial producer Halomonas elongata DSM $2581^{\top}$. Environ Microbiol .

42. Peter H, Weil B, Burkovski A, Krämer R, Morbach S: Corynebacterium glutamicum is equipped with four secondary carriers for compatible solutes: Identification, sequencing, and characterization of the proline/ ectoine uptake system, ProP, and the ectoine/proline/glycine betaine carrier, EctP. J Bacteriol 1998, 180:6005-6012.

43. Chen $\mathrm{CL}$, Beattie GA: Characterization of the osmoprotectant transporter OpuC from Pseudomonas syringae and demonstration that cystathionine- $\beta$-synthase domains are required for its osmoregulatory function. J Bacteriol 2007, 189:6901-6912.

44. Rabus R, Jack DL, Kelly DJ, Saier MH: TRAP transporters: an ancient family of extracytoplasmic solute-receptor-dependent secondary active transporters. Microbiology 1999, 145:3431-3445.

45. Jia Y, Yuan W, Wodzinska J, Park C, Sinskey AJ, Stubbe J: Mechanistic studies on class I polyhydroxybutyrate (PHB) synthase from Ralstonia eutropha: Class I and III synthases share a similar catalytic mechanism. Biochemistry 2001, 40:1011-1019.

46. Sohn SB, Kim TY, Park JM, Lee SY: In silico genome-scale metabolic analysis of Pseudomonas putida KT2440 for polyhydroxyalkanoate synthesis, degradation of aromatics and anaerobic survival. Biotechnol J 2010, 5:739-50

47. Cai L, Yuan MQ, Liu F, Jian J, Chen GQ: Enhanced production of mediumchain-length polyhydroxyalkanoates (PHA) by PHA depolymerase knockout mutant of Pseudomonas putida KT2442. Bioresour Technol 2009, 100:2265-2270.

48. York GM, Lupberger J, Tian J, Lawrence AG, Stubbe J, Sinskey AJ: Ralstonia eutropha $\mathrm{H} 16$ encodes two and possibly three intracellular poly[D-(-)-3hydroxybutyrate] depolymerase genes. J Bacteriol 2003, 185:3788-3794.

49. Goo YA, Roach J, Glusman G, Baliga NS, Deutsch K, Pan M, Kennedy S, DasSarma S, Ng WV, Hood L: Low-pass sequencing for microbial comparative genomics. BMC Genomics 2004, 5:3.

50. Bonneté F, Madern D, Zaccai G: Stability against denaturation mechanisms in halophilic malate-dehydrogenase "adapt" to solvent conditions. J Mol Biol 1994, 244:436-447.

51. Kennedy SP, Ng WW, Salzberg SL, Hood L, DasSarma S: Understanding the adaptation of Halobacterium species NRC-1 to its extreme environment through computational analysis of its genome sequence. Genome Res 2001, 11:1641-1650.

52. Maskow T, Babel W: Calorimetrically obtained information about the efficiency of ectoine synthesis from glucose in Halomonas elongata. Biochim Biophys Acta 2001, 1527:4-10.

53. Naughton LM, Blumerman SL, Carlberg M, Boyd EF: Osmoadaptation among Vibrio species and unique genomic features and physiological responses of Vibrio parahaemolyticus. Appl Environ Microbiol 2009, 75:2802-2810.

doi:10.1186/1475-2859-10-88

Cite this article as: Cai et al: Comparative genomics study of polyhydroxyalkanoates ( $\mathrm{PHA}$ ) and ectoine relevant genes from Halomonas sp. TD01 revealed extensive horizontal gene transfer events and co-evolutionary relationships. Microbial Cell Factories 2011 10:88. 